\title{
First-Principles studies of silicon underpotential deposition on defective graphene and its relevance for lithium-ion battery materials
}

\author{
M. Laura Urquiza ${ }^{a}$, Manuel Otero ${ }^{a, b}$, Guillermina L. Luque ${ }^{a, *}$, Daniel Barraco ${ }^{b}$, \\ Ezequiel P.M. Leiva ${ }^{a, *}$ \\ a INFIQC, Departamento de Matemática y Física, Facultad de Ciencias Químicas, Universidad Nacional de Córdoba, CONICET, Ciudad Universitaria, 5000 \\ Córdoba, Argentina \\ b IFEG, Facultad de Matemática Astronomía y Física, Universidad Nacional de Córdoba, CONICET, Ciudad Universitaria, 5000 Córdoba, Argentina
}

\section{A R T I C L E I N F O}

\section{Article history:}

Received 1 April 2016

Received in revised form 27 April 2016

Accepted 1 May 2016

Available online 4 May 2016

\section{Keywords:}

graphene

silicon

lithium-ion batteries

underpotential deposition

DFT

\begin{abstract}
A B S T R A C T
First-principles calculations are undertaken to analyze the properties of carbon-silicon hybrid materials consisting of silicon modified graphene and defective graphene to evaluate the stability of the structure and their interactions with lithium. Underpotential shifts are determined for the different structures on defective surfaces, showing that decoration of graphene defects with a small number of silicon atoms should occur at underpotentials. Nucleation overpotentials are also determined using a thermodynamic formalism, showing that the formation of nuclei should be hindered with respect to free standing Si clusters. These results analyzes the possibility of using underpotential deposition of silicon on graphene to obtain high capacity and cycling stable material for anodes of lithium batteries.
\end{abstract}

(c) 2016 Elsevier Ltd. All rights reserved.

\section{Introduction}

In terms of sustainable development and environmental issues, the use of efficient storage systems is an increasingly critical point. The world has undergone a great technological revolution in the past 20 years led by lithium ion batteries, due to their relatively high energy density. However, in order to meet the energy demand of our modern life, a new generation of lithium batteries, more powerful, efficient and cheaper, is desirable. The challenge lays in choosing electrochemically active, light, abundant and environmentally friendly materials that achieve high density energy [1-4], long life cycle and when possible, a high power density. Silicon has shown to be a very promising anode material to fulfill the needs of a lithium-ion battery. It has a high theoretical capacity, of around $3579 \mathrm{mAh} / \mathrm{g}$ [5], low cost and natural abundance. However this material runs with the great disadvantage that during the formation of a $\mathrm{Si}$-Li alloy corresponding to the insertion of $\mathrm{Li}$ in the anode for the charging process, it suffers a change of volume that can reach $380 \%$ [6-8]. This expansion, followed by contraction of the material upon battery discharge, quickly leads to irreversible damage of the electrode, causing a rapid decrease in capacity

\footnotetext{
* Corresponding authors.

E-mail addresses: gluque@fcq.unc.edu.ar (G.L. Luque), eleiva@fcq.unc.edu.ar (E.P.M. Leiva).
}

during cycling [9-11]. Furthermore, Si usually has a low electrical conductivity. In this regard, some current studies propose the combination of Si with graphene sheets to improve conductivity [12-15]. Even more, the combination of Si-based materials with carbonaceous materials as graphene can substantially relieve the mechanical stress suffered by the electrode [16]. Graphene has been proposed as one of the best candidate matrices for anodes due to its mechanical strength, high surface area, porous structure, and the capacity to provide a high flexibility to tolerate volume changes [17-19]. On the basis of these considerations, an appealing option appears to be the generation of graphene/Si multilayer structures at the nanometer scale, as proposed in the article of Ji et al. [15]. These authors have used a repeated process of filtering liquid-phase exfoliated graphene film and a subsequent coating of amorphous Si film via plasma-enhanced chemical vapor deposition method to get graphene/Si multilayer structures. However, the cycling performance did not fill the expectations that this material awaked. The SEM pictures of these compounds show that while the desired structures have been successfully obtained, the adhesion between the Si and C materials is relatively weak. These features can be understood in terms of the weak interaction between $\mathrm{Si}$ nanostructures and graphitic structures, as shown by first principles calculations. While the minimum binding energy of a single $\mathrm{Si}$ atom to a (0001) graphite surface is $E_{g r}^{S i}=-1.768 \mathrm{eV}$, the binding energy of Si clusters to the surface denotes a dramatically 
weaker interaction of $\mathrm{Si}$ with the graphite surface as cluster size increases. For example, the binding energy is $-0.13 \mathrm{eV} /$ atom for $\mathrm{Si}_{3}$ and $-0.055 \mathrm{eV} /$ atom for $\mathrm{Si}_{7}$. If we compare the $E_{g r}^{S i}$ value given above with the experimental bulk cohesive energy of $\mathrm{Si}, E_{\text {coh }}^{S i}=$ $-4.97 \mathrm{eV}$, we can understand the progressively weaker Si-graphite interaction in bond-order terms: as a Si atom finds more and more $\mathrm{Si}$ neighbors, its bond with the graphite surface becomes progressively weaker. This weak interaction between silicon nanostructures and graphite has been also confirmed by STM experiments [20]. A possible remedy to this weakened interaction could be sought by functionalizing the carbonaceous surface. Kulish et al. [21] have shown that functionalization with carboxyl groups of carbon nanotubes improved considerably the bonding of the Si clusters to the carbonaceous nanostructure. In fact, the presence of the - $\mathrm{COOH}$ groups was found to be able to strengthen the $\mathrm{Si}_{6}-(5,5)$ nanotube interaction in $-0.6 \mathrm{eV}$. Another way to improve the adhesivity between silicon and carbon could be the generation of silicon carbide at this interface through intermixing induced by Ar-ion beam [22]. We analyze here a different alternative to improve the stability the Si adsorbed structures: the introduction of surface defects.

In the present work we perform first-principle studies of carbon-Silicon hybrid materials consisting of silicon modified graphene and defective graphene to evaluate the stability of the structures and their interactions with lithium. We evaluate the effect of the presence of simple and double vacancy defects in the interaction with silicon and the interaction of these hybrids structures with lithium. We discuss this information in the context of underpotential deposition, which could be relevant to seek an electrochemical alternative for the controlled decoration of graphenic surfaces.

\section{Calculations Methods and Modeling}

\subsection{Calculation details}

The ab initio calculations were performed with the Quantum Espresso package [23], which is based on plane waves bases, setting up a kinetic energy cutoff of $680 \mathrm{eV}$. For the calculations we used ultrasoft pseudopotentials within the Perdew-Wang approximation for the exchange correlation functional in the PW91 functional $[24,25]$. The simulation box consisted in a $4 \times 4$ graphene supercell containing $32 \mathrm{C}$ atoms with periodic boundary conditions in all directions. The cell size was kept constant during optimization. In the $z$ direction we leave a $10 \AA ̊$ vacuum to avoid interaction among the graphene layers. In the case of larger clusters of silicon we used as a control a graphene supercell containing $60 \mathrm{C}$ atoms observing no significant differences with the results obtained for the one with $32 \mathrm{C}$ atoms. We evaluated pristine and defective graphene considering simple and double vacancy defects. The Brillouin zone was sampled in a $4 \times 4 \times 1$ irreducible Monkhorst-Pack k point grid [26]. The convergence threshold for the total energy at each electronic calculation was set to $1 \times 10^{-5} \mathrm{eV}$, taking into account Van der Waals interactions using the DFT-D method, since results for similar systems [27] have shown that is important to consider dispersion interactions to get a good accuracy in the energy values. Geometry optimizations were performed employing the Broyden-Fletcher-Goldfarb-Shanno (BFGS) algorithm (for stress minimization).

\subsection{Model for defective graphene and its decoration with Si atoms.} Thermodynamic analysis

The interaction of a silicon atom and silicon clusters with a pristine and a defective graphene surface was analyzed by the calculation of different energy-related quantities that are relevant to the present problem.

In order to analyze the stability of adsorbed $\mathrm{Si}$ atoms with respect to isolated $\mathrm{Si}$ atoms in the gas phase, it is useful to define a cluster formation energy referred to the atomic state as:

$\Delta E_{f o r}^{a t}=E_{S i_{N} G r}-E_{G r}-N E_{S i}$

where $E_{S_{N} G r}$ is the energy of the optimized hybrid structure (pristine or defective graphene, modified with $N$ silicon atoms), $E_{G r}$ is the energy of pristine or defective graphene and $E_{S i}$ is the energy of a silicon atom in vacuum. Thus, a negative value of $\Delta E_{f o r}^{a t}$ indicates that the Si adatoms are more stable in the nanostructure than in the gas phase.

Alternatively, in order to determine the relative stability of the surface Si nanostructures with respect to the bulk Si material it is useful to define the following cluster formation energy from the bulk material, denoted with:

$\Delta E_{f o r}^{b u l k}=E_{S i_{N} G r}-E_{G r}-N E_{S i B u l k}$

where $E_{\text {Sibulk }}$ is the cohesive energy of $\mathrm{Si}$ in diamond structure and $N$ the number of silicon atoms in the nanostructure. In this case, a negative value of the formation energy $\Delta E_{\text {for }}^{\text {bulk }}$ indicates a favorable formation of the hybrid structure from the bulk materials. As found below, the quantity $\Delta E_{\text {for }}^{\text {bulk }}$ is particularly useful in the case of electrochemistry, since it is closely related to the free energy of formation of the corresponding nanostructure, which can be used to predict its electrochemical stability. With this purpose, we shortly revisit the modeling developed by some of us with the purpose of analyzing the stability of metallic nanostructures deposited in a cavity of a foreign substrate[28]. Similar modeling
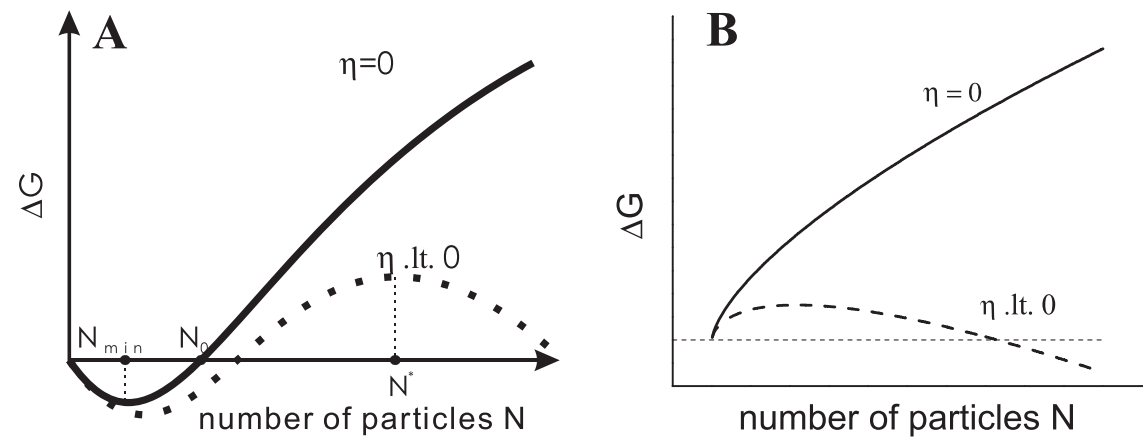

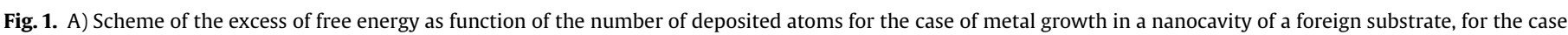

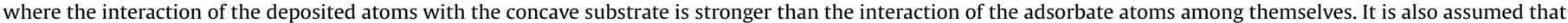

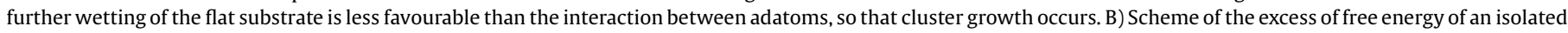
metal cluster as function of the number of atoms at two different overpotentials. 
was developed for metal deposition on a nanoparticle made of a different metal [29,30]. Fig. 1 (a) shows the qualitative shape of the excess of free energy for cluster formation as a function of the number of atoms $N$, expected for the growth of a nanostructure where the interaction with the substrate within the nanocavity is stronger than the interaction with itself, while the interaction with the substrate becomes unfavorable outside de cavity. It can be seen that negative excess free energy values are obtained for the decoration of the nanocavity, while then the free energy starts to increase as a consequence of the growth over the surface level. The excess of free energy for the formation of the nanostructure at the overpotential $\eta$ was defined in reference [28] as:

$\Delta G(N)=\left[\left(G^{M / S}-G_{S}\right)-N \mu_{M}\right]+N z e \eta$

where $G^{M / S}$ is the free energy of the nanostructure and it closest environment, $G_{S}$ is the free energy of this environment previous to the formation of the nanostructure, $\mu_{M}$ is the chemical potential of the atoms being deposited in the corresponding bulk material and $z$ is the valence of the ions being deposited. Thus, it must be emphasized that the terms inside brackets of Eq. (3) are equivalent to those in Eq. (2), with the difference that the former include also entropic contributions. We will come back to this point below in the discussion.

The behavior of Fig. 1(A) may be contrasted with the growth of a free-standing, pure metal cluster Fig. 1B), where the free energy increases monotonically with the number of atoms as a consequence of the increasing surface exposed. The application of a negative overpotential (accounted for by the second term in Eq. (3)) also acts differently in both cases. In the case of the homoatomic system, the negative overpotentials generate instability in the growing clusters: clusters larger than that corresponding to the maximum in $\Delta G$ will growth towards the bulk phase, smaller ones will dissolve. On the other hand, in the heteroatomic system, the local minimum may persist, due to the occurrence of a free energy barrier for cluster growth. This situation will persist until the barrier may be overcome due to thermal fluctuations.

The two different behaviors shown in Fig. 1 will be useful to interpret the results found in the calculations of $\mathrm{Si}$ deposition on graphene defects.

A third definition that will be used here is what we will call cluster adsorption energy $\Delta \mathrm{E}_{a d s}^{N}$, which we define as:

$\Delta \mathrm{E}_{a d s}^{N}=E_{S i_{x} G r}-E_{G r}-E_{S i}^{N}$

where $E_{S i}^{N}$ now denotes the energy of a Si cluster in vacuum, made of $N$ Si atoms. This quantity will be used to make an estimation of the adhesivity of the cluster to the graphenic surface.
In the case of a single $\mathrm{Si}$ atom adsorption on pristine graphene, we considered three different adsorption sites of high symmetry: on top of a carbon atom (top), at the midpoint of a carbon-carbon bond (bridge), and at the center of the hexagon defined by the carbon atoms (hollow). In the case of defective surfaces, the Si atom was placed at a simple (SV) and at a double vacancy (DV). The Si atoms either bonds with three other atoms in a single vacancy site or with four atoms in a double-vacancy site. These kinds of structures are the most common structures seen in the silicon modified graphene structures [31]. For the case of cluster adsorption the free energy per silicon atom is calculated as follows:

$\Delta E_{\text {for } / N}=\frac{\Delta E_{\text {for }}}{N}=\left(E_{S i_{N} G r}-E_{G r}-N E_{\text {Sibulk }}\right) / N$

\subsection{Li binding energy and lithiation potential}

On the charging process lithium ion are inserted in the anode, and the electrochemical lithiation can be ideally represented as,

$y \mathrm{Li}^{+}+\mathrm{Si}_{x} \mathrm{Gr}+y \mathrm{e}^{-} \leftrightarrow \mathrm{Li}_{y} S i_{x} G r$

In this respect, it is useful to refer the potential to the bulk lithium deposition potential. This reaction can be written as

$L i^{+}+e^{-} \leftrightarrow L i$

So, the lithiation potential $V_{\text {lit }}$ for the previous reaction can be calculated with the following equation

$V_{\text {lit }}=-\frac{\Delta G}{z F}$

where $\Delta G$ is the change in the Gibb's free energy per mol for $L i_{y} S i_{x} G r$ formation from bulk lithium and $S i_{x} G r, z$ is the number of electrons involved in the electrochemical reaction, in this case one, and $\mathrm{F}$ the Faraday constant. Neglecting entropy and pressure terms, we can approximate the change in formation Gibb's energy by the internal energy change, $\Delta G_{f} \cong \Delta E_{f}$, as obtained from DFT calculations [32]. This internal energy change for the formation of the lithiated structure is:

$\Delta E_{f}=E_{S i_{x} G r L i_{y}}-E_{S i_{x} G r}-y E_{\text {Libulk }}$

where $E_{S i_{x} G r L i_{y}}$ is the energy of the lithiated structure, $E_{S i_{x} G r}$ is the energy of the graphene layer modified with silicon, and $E_{\text {Libulk }}$ is the energy of a $\mathrm{Li}$ atom in the elemental body centered cubic $\mathrm{Li}$ structure. Negative values indicate thermodynamic preference for $\mathrm{Li}$ insertion while positive values indicate preference for bulk lithium formation. The $\Delta E_{f}$ value in $\mathrm{eV}$ can be related to the experimentally measured potential in volts for the occurrence of

Table 1

Formation energies calculated for the introduction of a silicon atom in/on a graphene layer and the optimized different structures.

\begin{tabular}{|c|c|c|c|c|c|}
\hline \multirow[t]{2}{*}{ System } & \multicolumn{3}{|c|}{ Graphene-Si } & \multirow[t]{2}{*}{ SV-Graphene-Si } & \multirow[t]{2}{*}{ DV-Graphene-Si } \\
\hline & Top & bridge & hollow & & \\
\hline$\Delta E_{\text {for }}^{a t}[\mathrm{eV}]$ & -1.09 & -1.15 & -0.55 & -8.86 & -8.81 \\
\hline$\Delta E_{\text {for }}^{\text {bulk }}[\mathrm{eV}]$ & 3.89 & 3.82 & 4.43 & -3.88 & -3.84 \\
\hline Structure & & & & & \\
\hline & & & 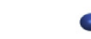 & & \\
\hline
\end{tabular}


LiGraphene-Si structure versus $\mathrm{Li}^{+} / \mathrm{Li}^{0}$ by the following equation [33]:

$V_{\text {lit }}=-\frac{\Delta E_{f}}{x e_{0}}$

where $e_{0}$ is the elementary charge, and $\mathrm{x}$ is the number of Li atoms involved in the electrochemical reaction.

\section{Results and Discussion}

\subsection{Formation energy of a single silicon atom on pristine and defective graphene}

In order to study the energetically most favored places to adsorb a silicon atom on a pristine graphene sheet, we placed the former on top, bridge, and hollow sites of the latter and compared these structures with defective graphene. Table 1 show the adsorption and formation energy of the different silicon modified structures together with the optimized geometries found in each case.

The results show that in the case of pristine graphene, the most stable structure is the one where the $\mathrm{Si}$ atom is placed on bridge of a $\mathrm{C}-\mathrm{C}$ bond, since this place presents the most negative adsorption energy, in agreement with literature results [34-36]. Nevertheless, the energy difference between the bridge and top structures is comparable with the room thermal energy ( $\mathrm{kT} \sim 0.03$ $\mathrm{eV}$ ), indicating that the diffusion of $\mathrm{Si}$ atoms is possible along the surface. It is interesting to notice that although all the adsorption energies are negative, the interaction between pristine graphene and the silicon atom is relatively weak as compared with the strong $\mathrm{sp}^{2}$ interaction among $\mathrm{C}$ atoms in the graphene lattice, producing no distortion of the graphene layer [37]. In this regard, we remind that the binding energy of $\mathrm{C}$ atoms in the graphene and related curved structures is between 7.35 and $7.70 \mathrm{eV} /$ atom [38]. For Si adsorption on pristine graphene, it can be noticed that the bulk formation energies $\Delta E_{\text {for }}^{\text {bulk }}$ are positive, indicating than the further addition of more silicon atoms on the surface would lead to their diffusion on the surface to join together and form silicon clusters, which as discussed in the introduction, show a poor adhesion to the graphenic surface. In all the cases the distance between $\mathrm{Si}-\mathrm{C}$ is $2.13 \AA$.

For the defective graphene structures, the single adatom values of $\Delta E_{f o r}^{\text {bulk }}$ are favorable. These results demonstrate that the two structures shown on the right of Table 1 should form at potentials more positive than the bulk Si deposition potential (underpotential region). In these cases, the silicon modification produces changes in the geometry of the defective graphene layer due to the stronger interaction. This is in agreement with experimental results, which show [39] that in the case of a simple vacancy defect, the atomic orbital of silicon atom displaces outwards from the graphene plane, exhibiting a $\mathrm{sp}^{3}$ hybridization corresponding to a tetrahedral geometry. On the other hand, in the double-vacancy defect the silicon atomic orbital shows an $\mathrm{sp}^{2} \mathrm{~d}$ hybridization, allowing the silicon atom to remain in the graphene plane, as can be seen in the Figures of Table 1, in agreement with other theoretical reports [40].

In accordance with experimental results obtained for graphene single-vacancy and double-vacancy defects, using scanning transmission electron microscope with simultaneous annular dark-field imaging by Zhou et al. [31], the Si atoms either bond with three other atoms in a single-vacancy site or four atoms in a double-vacancy site in the graphene lattice, as can be directly observed from the images reported there.

\subsection{Structure of the Density of States}

It is well known that pure bulk $\mathrm{Si}$ is a semiconductor, with a relatively large resistivity [41]. On the other hand, the density of states of graphene presents a minimum at the Fermi level [42]. Since the conductivity of composites in Li-ion batteries electrodes is an important issue, we analyze here the electronic properties of the hybrid systems presented in the previous section. With this purpose, we performed DOS calculations of the different graphene structures without and with silicon.

As it can be seen in Fig. 2A, the presence of a single $C$ atom vacancy breaks the symmetry of the system and creates states at the Fermi level (due to the dangling $\mathrm{sp}^{2}$ orbitals), which are not present in the pristine graphene DOS. When these structures are modified with a silicon atom the electronic states are strongly altered. When graphene is in the presence of one Si atom, the DOS shows new states at the Fermi level that are mainly due to the $3 p$ orbitals of $\mathrm{Si}$ atoms. In the case of SV-graphene, the Si states overlap with the DOS of the system at the Fermi level due to the strong interaction among silicon atom and its adjacent $\mathrm{C}$ atoms in the graphene layer. The $\mathrm{Si}$ atom replaces the $\mathrm{C}$ atom yielding three Si-C covalent bonds, resulting in a DOS similar to that of graphene, with a minimum at the Fermi Level. These results are in accordance with the experimental studies performed by Zhou et al. [31], where it was shown that $\mathrm{Si}$ atom adopts a $\mathrm{sp}^{3}$ hybridization in the case of single vacancy, being above the carbon atoms of the graphene layer, inducing a local curvature.

In the case of DV graphene, while the DOS without silicon is similar to pristine graphene, new states appear at the Fermi level when a silicon atom is present in this structure. These states, are dominated by the $3 \mathrm{p}$ atomic orbitals of $\mathrm{Si}$. This feature should make this structure conductive, once again due to the strong interaction between $\mathrm{C}$ and $\mathrm{Si}$ atoms. In all cases, the presence of $\mathrm{Si}$ alters the states mostly near the Fermi level, producing changes in the band gap of pristine and defective graphene.

When a Si atom is bonded to four atoms at a DV site in graphene, the Si 3d orbitals contribute significantly to the bonding, resulting in a planar $\mathrm{sp}^{2} \mathrm{~d}$-like hybridization, whereas threefold coordinated $\mathrm{Si}$ in graphene adopts the preferred $\mathrm{sp}^{3}$ hybridization. These features are also in agreement with the findings in the work of Zhou et al. [31].

The DOS of the graphenic system with a three atoms silicon cluster (not shown) results similar to that obtained with the single silicon atom (Fig. 2-B) adhered to the graphenic structure. Thus, it appears that the inclusion of more silicon atoms to the structure does not affect notably the electronic configuration. Similar kinds of structures have been obtained experimentally by producing vacancies in the graphene structure by chemical oxidation

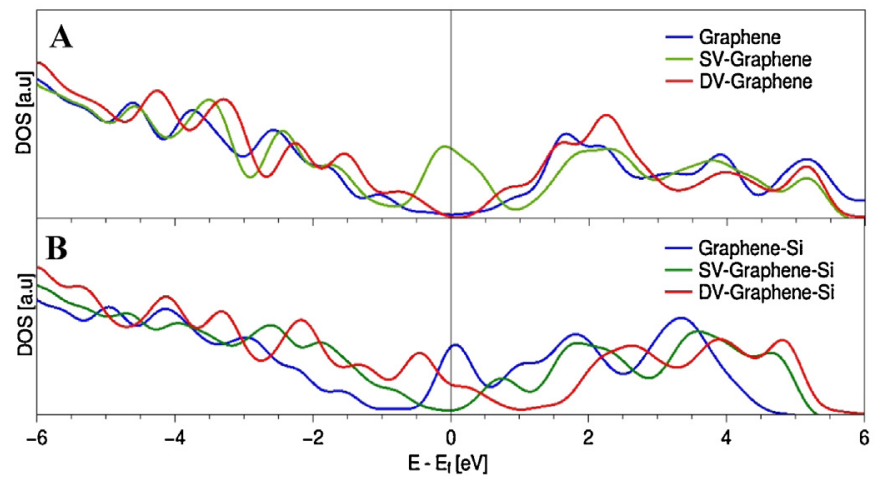

Fig. 2. Density of states of pristine and defective graphitic structures before (A) and after (B) modification with a silicon atom. Graphene (blue), SV-graphene (Green) and DV-graphene (red). 
methods and subsequent modification with silicon nanoparticles $[43,44]$.

\subsection{Difference charge analysis of the binding}

To understand the nature of the interaction between a deposited $\mathrm{Si}$ atom and the carbon atoms on the graphene layer, we study here the electronic density difference $\Delta \rho$ between the electron density of the pristine or defective graphene layer with silicon $\left(\rho_{S i G r}\right)$ and those of the separated pristine or defective graphene $\left(\rho_{G r}\right)$ and $\operatorname{Si}$ atom $\left(\rho_{S i}\right)$, at the optimized positions. Thus, $\Delta \rho$ is given by:

$\Delta \rho=\rho_{S i G r}-\rho_{G r}-\rho_{S i}$

The electron density differences obtained are depicted in Fig. 3 for the three systems considered here: $\mathrm{Si}$ on pristine graphene (A), Si on a single vacancy (B) and Si on a double vacancy (C)

In the case of pristine graphene, a pi-like interaction can be identified in Fig. $3 \mathrm{~A}$ between the silicon atom and the graphene layer. In fact, accumulation of electronic charge between the silicon atoms and the nearest $\mathrm{C}-\mathrm{C}$ atoms is found, with a nodal plane containing the interatomic bonds. The interaction is rather local, affecting only the two carbon atoms closer to the Si adsorbate.

For SV-graphene modified with one silicon atom, Fig. 3B, the different electron affinities of $\mathrm{Si}$ and $\mathrm{C}$ produce a change in the electron distribution of a wide region of the system, since the electrons are mainly localized within the bonds rather than mainly located on the $C$ atoms. The whole graphene structure remains covalent in nature, that is, there is an accumulation of charge between the three carbon atoms surrounding the silicon atom and the silicon atom. The electrons are mainly located on the carbon atoms rather than on the Si atom, this means that the electrons move from Si to neighboring carbon atoms, since $\mathrm{Si}$ is less electronegative than carbon. This results shows, in accordance with results from Tanga et al. [35], that Si can modify the electronic and chemical reactivity (see also DOS) of the graphene sheet, inducing a local charge redistribution. In the case of DV-graphene, Fig. 3C, there is a depletion of electrons from the silicon atom, which gives its electron to the dangling bonds of the carbon atoms forming covalent bonds with them, resulting in an accumulation of electrons between the $\mathrm{C}$ atoms and silicon. In the last two cases it can be seen that the interaction of Si with the carbon atoms of the defective graphene layer is a long ranged interaction, since $C$ atoms that are not directly attached to silicon suffer changes in their electronic structure. On the contrary, in the case of pristine graphene, the interaction among $\mathrm{C}$ atoms of the layer and the silicon atom is only local.

\subsection{Stability of small Si clusters on defective graphene}

To emulate the Si deposition process, we also studied the effect of the incorporation of more silicon atoms in the surface structures. In the case of pristine graphene, concomitant with the incorporation of more silicon atoms, there is an increment in the C-Si distance up to $4.36 \AA$ for the cluster with three silicon atoms, larger than the one observed for two and one silicon atoms (3.04 and $2.13 \AA$, respectively), in accordance with previous studies of Wu et al. [45] and M. Li et al. [27]. In the case of graphene, the $\mathrm{Si}_{3}$ cluster presents a shape of an isosceles triangle with $\mathrm{Si}-\mathrm{Si}$ bond length of $2.3 \AA$ in accordance with previous studies [27]. In the optimized structure, the $\mathrm{Si}_{3}$ cluster adsorbs in a parallel configuration to the graphene sheet, where two silicon atoms arrange in a bridge of a $\mathrm{C}-\mathrm{C}$ bond, which is the place where they present the highest energy adsorption.

According to the results shown in Table 2 the formation energy per $\mathrm{Si}$ atom diminishes (in absolute value) for the cluster with respect to the silicon atom (Table 1 ). This is in agreement with the results found by $\mathrm{Wu}$ et al. [45], who found that the adsorption energy decreases when the amount of silicon atom increases. Nevertheless for the defective graphene, this formation energy is still favorable; demonstrating that these structures of silicon clusters in a simple and double vacancy will form at potentials more positive than bulk silicon deposition. These results demonstrate the importance of the presence of defects for the formation of the hybrid silicon-carbon structures. As observed in the case of a single $\mathrm{Si}$ atom, the strong interaction between $\mathrm{Si}$ and $\mathrm{C}$ atoms produces a large distortion in the layers for single and double vacancy defects. In the case of defective layers, one Si atom strongly interacts with the carbon atoms of the graphene layer while the other two remain above the layer at a distance typical of a $\mathrm{Si}-\mathrm{Si}$ bond, that is $2.3 \AA$.

The different formation energies defined in Section 2.2 were analyzed for clusters of different sizes adsorbed on defective graphene with a double vacancy, as shown in Table 3 where two more columns have been added. The sixth column corresponds to the formation of a free-standing Si cluster from isolated atoms. That is, the energy is calculated according to Eq. (1), but in the absence of the graphene layer. Similarly, the seventh column considers the formation of a free standing cluster from bulk Si atoms, using Eq. (2) without the graphene layer.

A number of interesting conclusions may be drawn from this table, as follows:

- Within the cluster size considered, the formation energy $\Delta E_{f o r}^{a t}$ of the cluster on defective graphene starting from free Si atoms continuously decreases as a function of the number of atoms
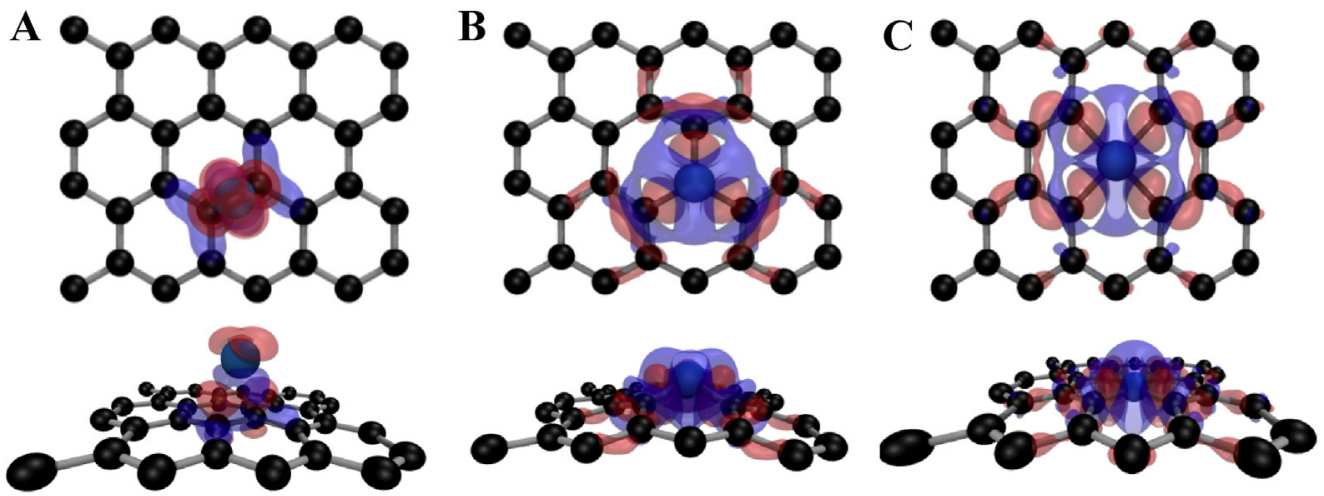

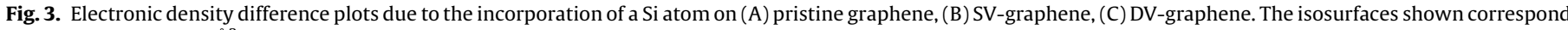
to a value of $0.0028 \mathrm{e} / \AA^{3}$. Red and blue colors indicate electronic charge accumulation and depletion respectively. Black: C-lattice; Blue spheres: Si atom. 
Table 2

Formation energies calculated and optimized configurations for the introduction of silicon clusters $\mathrm{Si}_{3}$ in the different structures.

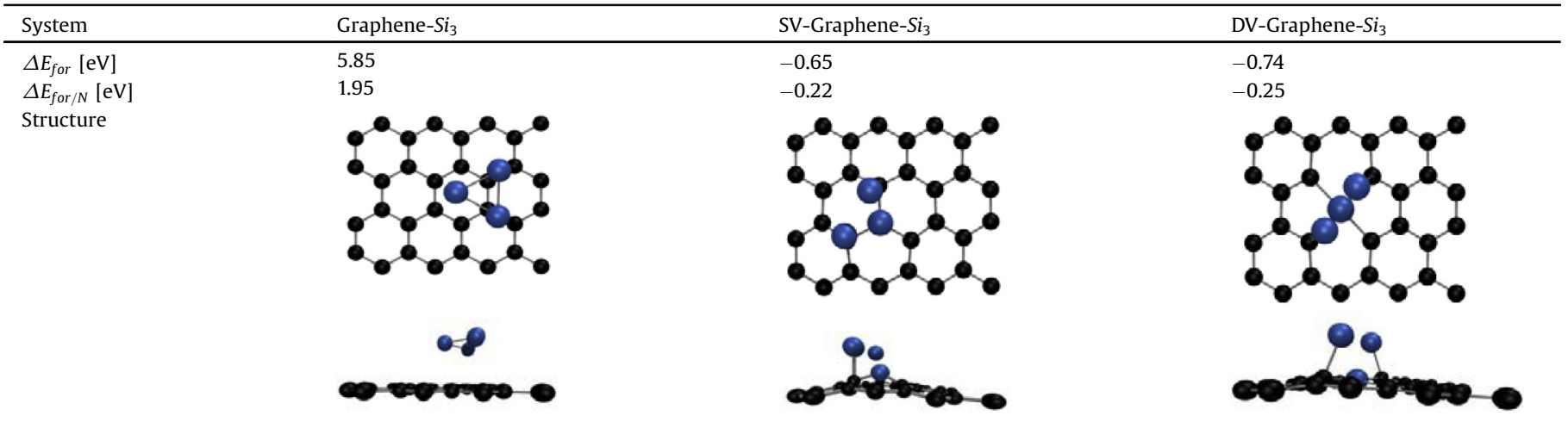

Table 3

Values of the different formation energies of cluster as defined in the text. The calculated bulk cohesive energy of Si is $-4.97 \mathrm{eV}$.

\begin{tabular}{|c|c|c|c|c|c|c|}
\hline \# Silicon atoms in cluster & $\Delta E_{\text {for }}^{a t}(\mathrm{eV})$ & $\Delta E_{\text {for }}^{\text {bulk }}$ & $\Delta E_{f o r / N}$ & $\Delta \mathrm{E}_{a d s}^{N}$ & $\Delta E_{f o r, v a c}^{a t}$ & $\Delta E_{f o r, v a c}^{\text {bulk }}$ \\
\hline 1 & -8.81 & -3.84 & -3.84 & -8.81 & 0 & 4.97 \\
\hline 2 & -12.53 & -2.59 & -1.30 & -8.43 & -4.1 & 5.84 \\
\hline 3 & -15.66 & -0.65 & -0.25 & -7.13 & -8.53 & 6.38 \\
\hline 4 & -19.886 & 0.00 & -0.001 & -6.55 & -13.34 & 6.54 \\
\hline 5 & -24.35 & 0.50 & 0.10 & -6.46 & -17.85 & 7 \\
\hline 6 & -28.77 & 1.05 & 0.18 & -5.64 & -22.56 & 7.26 \\
\hline 7 & -32.7 & 2.09 & 0.30 & -6.07 & -27.23 & 7.56 \\
\hline
\end{tabular}

(second column), with an energy increment of -4.4 to $-4.8 \mathrm{eV}$ per added atom.

- The largest difference between columns 2 and 6 is found for $N=1$, corresponding to the adsorption of a single atom on the defective graphene with an energy difference of $-8.81 \mathrm{eV}$, decreasing this difference down to $-6.07 \mathrm{eV}$ for $\mathrm{N}=7$. This means that as the cluster becomes larger, it is stabilized to a smaller extent by the presence of the defect.

- In fact, the differences between columns 2 and 6 correspond to the adsorption energy of the cluster, as defined in Eq. (4), reported on column (5) of Table 3 and plotted in Fig. 4. From this figure, it appears that as the cluster becomes larger, its binding energy to the defect approaches a limiting value around $-6.0 \mathrm{eV}$. Assuming a binding energy in this range, we can estimate the number of defects that would be required to have a good adhesion of the Si structures to graphene. If we consider the adhesion between graphene layers in graphite as satisfactory, we can make this estimation. Both experiment [46] and theory [47] indicate that the adhesion between graphite layers is about $0.31 \mathrm{~J} / \mathrm{m}^{2}\left(0.019 \mathrm{eV} / \AA^{2}\right)$. Using the present figures, it appears that the occurrence of a double vacancy defect (filled by $\mathrm{Si}$ ) every $300 \AA^{2}$ would be enough to provide a good adhesion between graphite and $\mathrm{Si}$, equivalent to that existing between graphite layers.

- The values of $\Delta E_{\text {for }}^{\text {bulk }}$ in column 3 indicate that the formation of up to 3 atoms Si clusters will be, from an energetic viewpoint, favorable at underpotentials. This is illustrated more clearly in

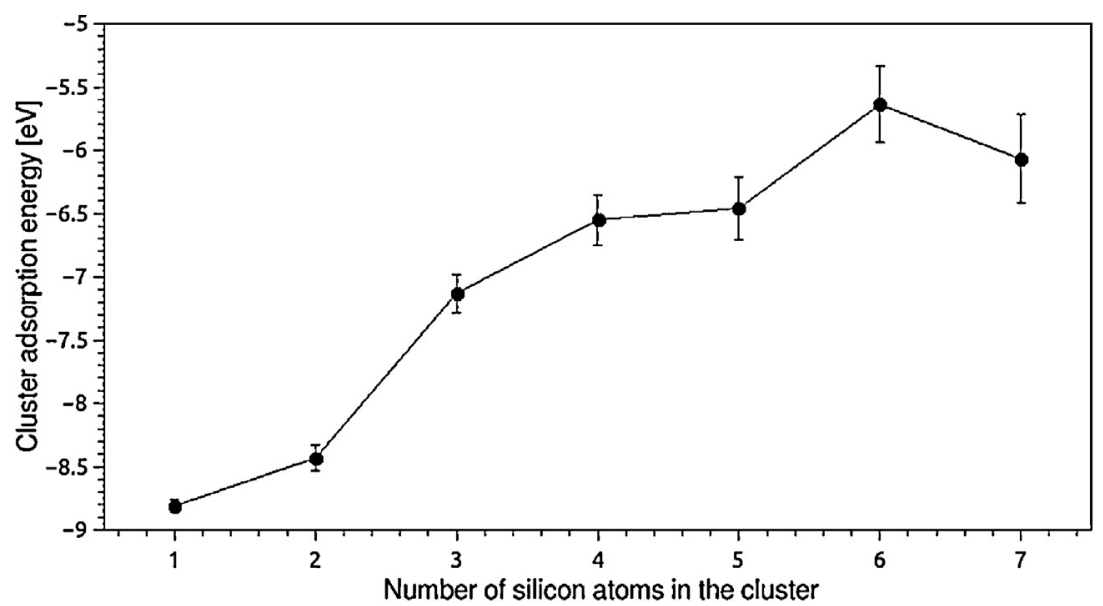

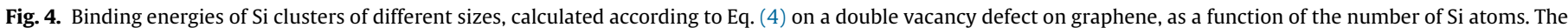

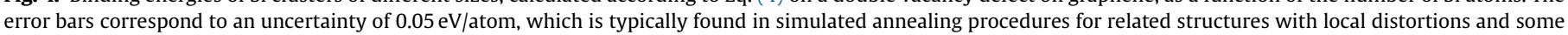
bond switching [49]. 


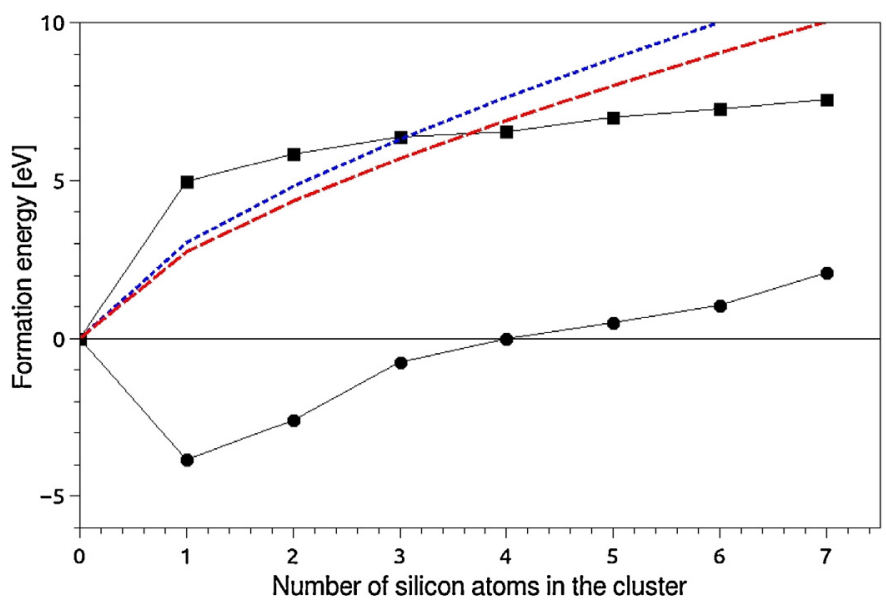

Fig. 5. (-) Formation energy of a Si cluster with different number of atoms on a double vacancy defect, defined according to Eq. (2). ( $\boldsymbol{\square}$ ) Formation energy of a freestanding Si cluster in vacuum with different number of atoms. (dotted blue curve) Formation energy of clusters calculated using the surface energy of the $\mathrm{Si}(100)$ surface(broken red curve) Formation energy of clusters calculated using the surface energy of $\mathrm{Si}(111)$ surface.

Fig. 5. The curve shown there in filled circles corresponds to the quantity defined in Eq. (2), which as discussed above corresponds to Eq. (3) for $\eta=0$ without accounting for entropic contributions, which are usually small for the present systems [48]. We can see that this curve resembles the qualitative behavior of the full line in Fig. 1(a), in the sense that at $\eta=0$ there are several nanostructures that present $\Delta G(N)<0$, indicating that they would survive in the underpotential (upd) region. Using equation with $N=1$ and $z=4$, we see that the decoration of DV defects with a single $\mathrm{Si}$ atom will be possible at underpotentials of $\eta^{\text {upd }}=0.96 \mathrm{~V}$. Si upd decoration still remains favorable for $N=2\left(\eta^{\text {upd }}=0.65 \mathrm{~V}\right)$ and $N=3\left(\eta^{\text {upd }}=0.16 \mathrm{~V}\right)$ but the formation of Si clusters with $N=4$ or larger would involve getting into the bulk Si deposition region. It is expected that in the presence of graphene with larger defects, upd would be favorable for clusters with more silicon atoms.

- Column 7 in Table 3 presents the formation energies $\Delta E_{\text {for,vac }}^{\text {bulk }}$ of free standing clusters from the bulk Si materials, which are plotted in Fig. 5 as filled squares. In this case, we see that the behavior of $\Delta E_{\text {for } v \text { vac }}^{\text {bulk }}$ resembles that of the full line of Fig. $1 \mathrm{~B}$, presenting a monotonic growth with the number of particles. For comparison, we present also in Fig. 5 the formation energy of clusters calculated assuming a spherical shape for the cluster and using the surface energy of the $\mathrm{Si}(100)$ [49] (dotted blue curve) and $\mathrm{Si}(111)$ faces (broken red curve) [50]. It is found that the calculation assuming a smooth, spherical cluster, present a faster change with the number of atoms than the atomistic calculation.

- The data in column 3 of Table 3 may be used along with Eq. (3) to estimate the nucleation overpotential for different clusters sizes. Taking the derivative of this equation and equaling it to 0 , allows to determine the critical potential $\eta_{\text {crit }}$ at which nuclei of different sizes will become critical [51]:

$$
\begin{aligned}
\eta_{\text {crit }}\left(N^{*}\right) & =-\left.\frac{1 d}{z e_{0} d N}\left[\left(G^{M / S}-G_{S}\right)-N \mu_{M}\right]\right|_{N=N^{*}} \\
& \approx-\left.\frac{1 d}{z e_{0} d N}\left[\Delta E_{f o r}^{\text {bulk }}\right]\right|_{N=N^{*}} \text { emphasizing that the }
\end{aligned}
$$

where we $e_{0} d N$ emphasizing that the second equality is approximate since entropic contributions are neglected. Assuming $z=4$ we get values of $\eta_{\text {crit }}$ between $0.16 \mathrm{~V}$ and $0.26 \mathrm{~V}$ of $N^{*}$ ranging between 4 and 7 .

- If an analysis similar to the previous item is made for free standing clusters (using data in column 7 of table Table 3 ), it is found that the $\eta_{\text {crit }}$ falls to about $0.08 \mathrm{~V}$ for $N^{*}$ ranging between 4 and 7. That is, although the presence of defects enhances $\mathrm{Si}$ electrosorption, making it possible at underpotentials, it demands larger overpotentials to make a given cluster size critical.

\subsection{Properties of lithiated structures}

We now come to evaluate the interaction of small silicon clusters on graphene with lithium to estimate whether this structures may be good for their use as anodes for lithium-ion batteries. To do so, we take in account the values of formation energy of lithium in the silicon modified graphene structures. As previously mentioned, it is experimentally observed that graphene-silicon hybrid composites show great promise as anode materials, since the presence of both of them can enhance the ultimate material leading to better performances. We only focus on one $\mathrm{Si}$ atom and on the $\mathrm{Si}_{3}$ cluster, since according to Table 3 it is up to this amount of silicon atoms that the formation energy is favorable at underpotentials for double vacancy defects. Table 4 shows the lithiation potential obtained according to Eq. (8) for one and three silicon clusters

It is interesting to note that the lithiation potential on graphene in the absence of a $\mathrm{Si}$ atom delivers a negative value of $-0.6 \mathrm{~V}$ (not shown in the table), while in the presence of Si this potential $\left(V_{\text {lit }}=-\Delta E_{f} / \mathrm{xe}_{0}\right)$ is more positive $(-0.05 \mathrm{~V})$. In the presence of more silicon atoms the situation becomes even more favorable (for $\mathrm{Si}_{3} V_{\text {lit }}=1.09 \mathrm{~V}$ ). The latter value is also more favorable than the one observed for the interaction of the $\mathrm{Si}_{3}$ cluster with lithium on its

Table 4

\begin{tabular}{|c|c|c|c|c|c|c|}
\hline System & Graphene-Si-Li & SV-Graphene-Si-Li & DV-Graphene-Si-Li & Graphene- $S i_{3}-L i$ & SV-Graphene- $S i_{3}-L i$ & DV-Graphene- $\mathrm{Si}_{3}-\mathrm{Li}$ \\
\hline $\begin{array}{l}V_{\text {lit }}(\mathrm{V}) \\
\text { structure }\end{array}$ & -0.05 & 0.05 & 0.66 & 1.09 & $\begin{array}{r}0.93 \\
0\end{array}$ & 1.32 \\
\hline
\end{tabular}

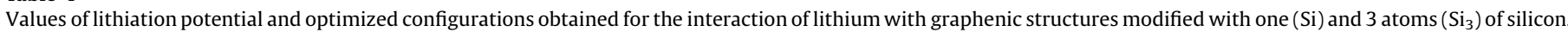


own $\left(V_{\text {lit }}=0.92 \mathrm{~V}\right)$. These results indicate that the hybrid material made of graphene and silicon enhances the interaction with lithium, providing additional intercalation sites. From the figure on the first column of Table 4 it is found that the adsorption of the lithium atom occurs at the center of the carbon hexagon close to $\mathrm{Si}$, at a distance $\mathrm{Si}-\mathrm{Li}$ of $2.65 \AA$. Although the values of lithiation potential are favorable for this structure, it is important to note that if we take a look at the values of formation energies of structures made of pristine graphene modified with one Si atom (Table 1 , column 1) and $\mathrm{Si}_{3}$ clusters (Table 2, column 1), we find that these structures should not form from a thermodynamic viewpoint. On the other hand, in the case of SV and DV-defective graphene sheets, not only the lithiation potential shows positive values for $\mathrm{Si}_{1}$ and $\mathrm{Si}_{3}$ indicating a favorable reaction (Table 4 , columns 2 and 3 for $\mathrm{Si}_{1}$ and columns 5 and 6 for $\mathrm{Si}_{3}$ ), but it is found that the graphenemodified silicon structures will also form, according to the formation values shown in Tables 1 and 2. For these defective structures, the lithium atom reaches the minimum energy when is located at a distance of around $2.7 \AA$ from the silicon atom, in accordance with the distance found in $\mathrm{Li}_{x} \mathrm{Si}$ alloys [52]. It is important to notice here that the interaction of lithium found in presence of silicon for these defective graphene structures is stronger than in its absence. Even more, from the results presented in Table 4, it can be concluded that the structures that present the larger values of lithiation potential are the ones of the double vacancy type. Thus, it can be stated that the presence of double vacancy defects not only promotes the formation of stable hybrid graphene-Si (with up to three atoms of $\mathrm{Si}$ ) structures, but also favors the lithiation process, which is more favorable in presence of more Si atoms.

In order to understand the nature of the bonding between lithium and the graphene sheet modified with silicon, we performed electronic density difference $\Delta \rho$ plots of the of the lithiated pristine or defective graphene layer modified with silicon. The latter quantity is defined as:

$\Delta \rho=\rho_{\text {SiGrLi }}-\rho_{\text {SiGr }}-\rho_{L i}$

where $\rho_{\text {SiGrLi }}$ is the electronic density of the pristine or defective layer with silicon and lithium, $\rho_{S i G r}$ is the electronic density of the system without the Li atom and $\rho_{L i}$ is the electronic density of the isolated lithium atom. The results for $\Delta \rho$ can be found in Fig. 6 for the case of pristine (Fig. 6A), simple vacancy (Fig. 6B), and double vacancy graphene sheets (Fig. 6C).
An important change in the electronic density plot can be observed in the presence of lithium. An accumulation of charge is noticeable between the $\mathrm{Li}$ and the $\mathrm{Si}$ atoms, at the expenses of charge depletion between the $\mathrm{Si}$ and $\mathrm{C}$ atoms. This feature suggests that the insertion of lithium would produce a weakening on the Si$\mathrm{C}$ interaction. It is also remarkable that the lithium atoms have a depletion of charge having a cationic nature with a covalent bond to the silicon atom and an ionic bond with the graphene.

\section{Conclusions}

The application of first-principles calculations to study the deposition of Si onto pristine and defective graphite layers leads to a number of interesting conclusions, which are not only important for the deposition of this element itself, but have consequences for its application in systems related to lithium-ion batteries. The main points that may be emphasized are:

- The binding of Si clusters to defective graphite surfaces shows to be very strong, amounting several eVs/cluster. This binding energy, of covalent type, could contribute to stabilize the interaction between $\mathrm{Si}$ nanostructures and graphene layers, thus being very benefitial to stabilize composites used to store lithium-ions. A rough estimation shows that the occurrence of a double vacancy defect on the graphene surface every $300 \AA^{2}$ would be enough to provide an adhesion between $\mathrm{Si}$ and graphite as strong as the adhesion between graphite layers.

- In the case of the incorporation of Si atoms into double vacancies that is analyzed in detail here, it is found that the Si atoms increase the density of states at the Fermi level, thus improving the conductivity of the system, and probably its catalytic activity.

- Double vacancy defects could be decorated at underpotentials with one, two and three Si atoms. While this could be envisaged as an advantage to decorate the graphenic surface, it may be represent a problem when side reactions (i.e. with the solvent) make take place. This fact must be related to the increase of the density of states at the Fermi level pointed out in the previous item.

- The overpotentials found for building the small clusters analyzed here on the graphene defective surface are relatively large, of the order of $200 \mathrm{mV}$, as compared with the corresponding overpotentials required to make free standing clusters of similar sizes critical.
A
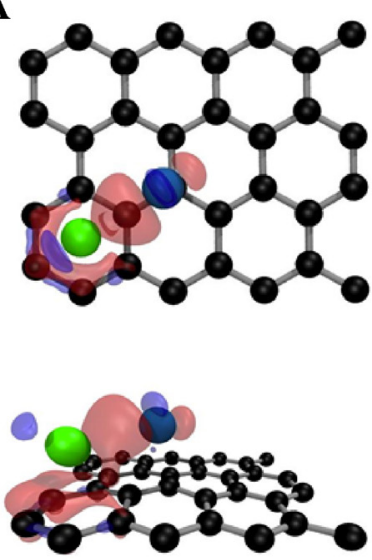

B
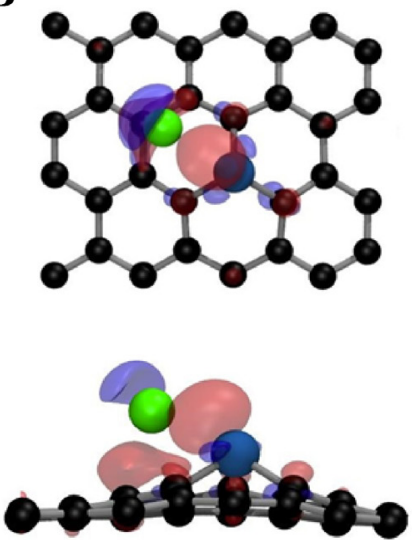

C
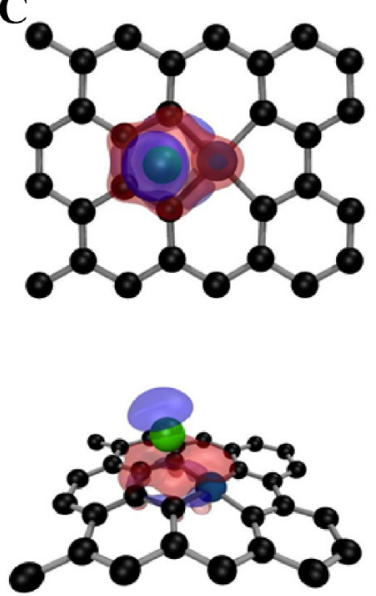

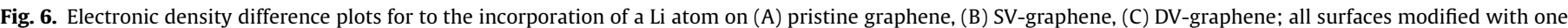

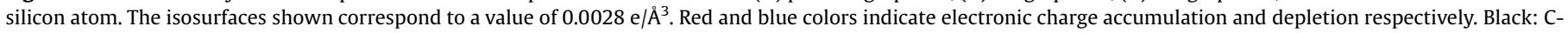
lattice; Blue spheres: Si atom; Green spheres: Li atom. 
- The lithiation potential is more favourable for the hybrid material graphene-Si than for the separated materials, and increases as the amount of silicon atoms rises. The hybrid material privides additional intercalation sites for lithium and would also, in principle, tolerate the volume changes suffered by silicon materials.

- The presence of defects not only enhances the interaction with silicon atoms but also favors the lithiation process, being this more favorable for the double vacancy defects.

\section{Acknowledgments}

This work was supported by grants PIP-11220110100992 and 11220150100624-CONICET, FONCYT PICT-2010-2324, SECyT Universidad Nacional de Córdoba, CCAD-UNC and GPGPU Computing Group. Support by Y-TEC, Argentina is also gratefully acknowledged.

\section{References}

[1] B. Diouf, R. Pode, Potential of lithium-ion batteries in renewable energy, Renew. Energy. 76 (2015) 375-380, doi:http://dx.doi.org/10.1016/j. renene.2014.11.058.

[2] D. Miranda, C.M. Costa, S. Lanceros-Mendez, Lithium ion rechargeable batteries: State of the art and future needs of microscopic theoretical models and simulations, J. Electroanal. Chem. 739 (2015) 97-110, doi:http://dx.doi. org/10.1016/j.jelechem.2014.12.010.

[3] J.B. Goodenough, K.-S. Park, The Li-ion rechargeable battery: a perspective, J. Am. Chem. Soc. 135 (2013) 1167-1176, doi:http://dx.doi.org/10.1021/ ja3091438.

[4] L. Mohammadzadeh, A. Goduljan, F. Juarez, P. Quaino, E. Santos, W. Schmickler, Nanotubes for charge storage - towards an atomistic model, Electrochim. Acta. 162 (2015) 11-16, doi:http://dx.doi.org/10.1016/j.electacta.2014.12.031.

[5] M.N. Obrovac, L. Christensen, Structural Changes in Silicon Anodes during Lithium Insertion/Extraction, Electrochem. Solid-State Lett. 7 (2004) A93-A96, doi:http://dx.doi.org/10.1149/1.1652421.

[6] J.M. Chem, N. Choi, Y. Yao, J. Cho, One dimensional Si/Sn - based nanowires and nanotubes for lithium-ion, J. Mater. Chem. 21 (2011) 9825-9840, doi: http://dx.doi.org/10.1039/c0jm03842c.

[7] L. Xue, G. Xu, Y. Li, S. Li, K. Fu, Q. Shi, et al., Carbon-Coated Si Nanoparticles Dispersed in Carbon Nanotube Networks As Anode Material for Lithium-Ion Batteries, Appl. Mater. Interfaces. 5 (2013) 21-25, doi:http://dx.doi.org/ 10.1021/am3027597.

[8] U. Kasavajjula, C. Wang, A.J. Appleby, Nano- and bulk-silicon-based insertion anodes for lithium-ion secondary cells, J. Power Sources. 163 (2007) 10031039, doi:http://dx.doi.org/10.1016/j.jpowsour.2006.09.084.

[9] B. Liang, Y. Liu, Y. Xu, Silicon-based materials as high capacity anodes for next generation lithium ion batteries, J. Power Sources. 267 (2014) 469-490, doi: http://dx.doi.org/10.1016/j.jpowsour.2014.05.096.

[10] X. Su, Q. Wu, J. Li, X. Xiao, A. Lott, W. Lu, et al., Silicon-Based Nanomaterials for Lithium-Ion Batteries: A Review, Adv. Energy Mater. 4 (2013) 1-23, doi:http:// dx.doi.org/10.1002/aenm.201300882.

[11] Y. Yao, M.T. Mcdowell, I. Ryu, H. Wu, N. Liu, L. Hu, et al., Interconnected Silicon Hollow Nanospheres for Lithium-Ion Battery Anodes with Long Cycle Life, Nano Lett. 11 (2011) 2949-2954, doi:http://dx.doi.org/10.1021/nl201470j.

[12] Y. Ye, X. Xie, J. Rick, F. Chang, B. Hwang, Improved anode materials for lithiumion batteries comprise non-covalently bonded graphene and silicon nanoparticles, J. Power Sources 247 (2014) 991-998, doi:http://dx.doi.org/ 10.1016/j.jpowsour.2013.08.048.

[13] W. Sun, R. Hu, H. Liu, M. Zeng, L. Yang, H. Wang, et al., Embedding nano-silicon in graphene nanosheets by plasma assisted milling for high capacity anode materials in lithium ion batteries, J. Power Sources 268 (2014) 610-618, doi: http://dx.doi.org/10.1016/j.jpowsour.2014.06.039.

[14] C. Chou, G.S. Hwang, Role of Interface in the Lithiation of Silicon- Graphene Composites: A First Principles Study, J. Phys. Chem. 117 (2013) 9598-9604, doi: http://dx.doi.org/10.1021/jp402368k.

[15] L. Ji, H. Zheng, A. Ismach, Z. Tan, S. Xun, E. Lin, et al., Graphene/Si multilayer structure anodes for advanced half and full lithium-ion cells, Nano Energy 1 (2012) 164-171, doi:http://dx.doi.org/10.1016/j.nanoen.2011.08.003.

[16] X. Yang, Z. Wen, X. Xu, B. Lin, Z. Lin, High-Performance Silicon/Carbon/Graphite Composites as Anode Materials for Lithium Ion Batteries, J. Electrochem. Soc. 153 (2006) 1341-1344, doi:http://dx.doi.org/10.1149/1.2199435.

[17] Z. Wu, G. Zhou, L. Yin, W. Ren, F. Li, H.-M. Cheng, Graphene/metal oxide composite electrode materials for energy storage, Nano Energy. 1 (2012) 107131, doi:http://dx.doi.org/10.1016/j.nanoen.2011.11.001.

[18] J. Ren, O. Wu, G. Hong, W. Zhang, H. Wu, K. Amine, et al., Silicon-Graphene Composite Anodes for High-Energy Lithium Batteries, Energy Technol. 1 (2013) 77-84, doi:http://dx.doi.org/10.1002/ente.201200038.
[19] F. Maroni, R. Raccichini, A. Birrozzi, G. Carbonari, R. Tossici, F. Croce, et al., Graphene/silicon nanocomposite anode with enhanced electrochemical stability for lithium-ion battery applications, J. Power Sources. 269 (2014) 873-882, doi:http://dx.doi.org/10.1016/j.jpowsour.2014.07.064.

[20] B. Scheier, Films of silicon nanoparticles grown by gas aggregation, J. Appl Phys. 94 (2003) 6069-6075, doi:http://dx.doi.org/10.1063/1.1618936.

[21] V.V. Kulish, M. Ng, O.I. Malyi, P. Wu, Z. Chen, Improved binding and stability in Si/CNT hybrid nanostructures via interfacial functionalization: a firstprinciples study, RSC Adv. 3 (2013) 8446-8453, doi:http://dx.doi.org/10.1039/ c3ra40340h.

[22] F. Klauser, R. Stijepovic, N. Endstrasser, S. Jaksch, N. Memmel, P. Scheier Oxidation study of silicon nanoparticle thin films on HOPG, Surf. Sci. 603 (2009) 2999-3004, doi:http://dx.doi.org/10.1016/j.susc.2009.08.007.

[23] P. Giannozzi, S. Baroni, N. Bonini, M. Calandra, R. Car, C. Cavazzoni, et al., QUANTUM ESPRESSO: a modular and open-source software project for quantum simulations of materials, J. Phys. Condens. MATTER. 21 (2009) 1-19, doi:http://dx.doi.org/10.1088/0953-8984/21/39/395502.

[24] C.F.P. John. Perdew, J.A. Chevary, S.H. Vosko, A. Koblar, Jackson Mark, R. Pederson, D.J. Singh, Carlos Fiolhais, Atoms, molecules, solids, and surfaces: Applications of the generalized gradient approximation for exchange and correlation, Phys. Rev. B 46 (1992) 6671-6687, doi:http://dx.doi.org/10.1103/ PhysRevB.46.6671.

[25] J.P. Perdew, Y. Wang, Accurate and simple analytic representation of the electron-gas correlation energy, Phys. Rev. B. 45 (1992) 244-249, doi:http://dx doi.org/10.1103/PhysRevB.45.13244.

[26] H.J. Monkhorst, J.D. Pack, Special points for Brillouin-zone integrations, Phys Rev. B. 13 (1976) 5188-5192, doi:http://dx.doi.org/10.1103/PhysRevB.13.5188.

[27] M. Li, Y. Liu, J. Zhao, X. Wang, Si clusters/defective graphene composites as Liion batteries anode materials: A density functional study, Appl. Surf. Sci. 345 (2015) 337-343, doi:http://dx.doi.org/10.1016/j.apsusc.2015.03.144.

[28] N.B. Luque, L. Reinaudi, P. Serra, E.P.M. Leiva, Electrochemical deposition on surface nanometric defects: Thermodynamics and grand canonical Monte Carlo simulations, Electrochim. Acta. 54 (2009) 3011-3019, doi:http://dx.doi. org/10.1016/j.electacta.2008.12.013.

[29] M.M. Mariscal, O.A. Oviedo, E.P.M. Leiva, On the selective decoration of facets in metallic nanoparticles, J. Mater. Res. 27 (2012) 1777-1786, doi:http://dx.doi. org/10.1557/jmr.2012.132.

[30] O.A. Oviedo, P. Vélez, V.A. Macagno, E.P.M. Leiva, Surface Science Underpotential deposition: From planar surfaces to nanoparticles, Surf. Sci. 631 (2015) 23-34, doi:http://dx.doi.org/10.1016/j.susc.2014.08.020.

[31] W. Zhou, M.D. Kapetanakis, M.P. Prange, S.T. Pantelides, S.J. Pennycook, J. Idrobo, Direct Determination of the Chemical Bonding of Individual Impurities in Graphene, Phys. Rev. Lett. 109 (2012) 1-5, doi:http://dx.doi.org/10.1103/ PhysRevLett.109.206803.

[32] C.B. Robledo, M. Otero, G. Luque, O. Cámara, D. Barraco, M.I. Rojas, et al., Firstprinciples studies of lithium storage in reduced graphite oxide, Electrochim. Acta. 140 (2014) 232-237, doi:http://dx.doi.org/10.1016/j. electacta.2014.07.013.

[33] X.H. Zhu, N. Chen, F. Lian, Y.P. Song, Y. Li, First principle calculation of lithiation delithiation voltage in Li-ion battery materials, Chinese Sci. Bull. 56 (2011) 3229-3232, doi:http://dx.doi.org/10.1007/s11434-011-4705-7.

[34] J. Wu, F. Hagelberg, K. Sattler, First-principles calculations of small silicon clusters adsorbed on a graphite surface, Phys. Rev. B 72 (2005) 085441, doi: http://dx.doi.org/10.1103/PhysRevB.72.085441.

[35] Y. Tang, Z. Liu, X. Dai, Z. Yang, W. Chen, D. Ma, et al., Theoretical study on the Sidoped graphene as an efficient metal-free catalyst for CO oxidation, Appl. Surf. Sci. 308 (2014) 402-407, doi:http://dx.doi.org/10.1016/j.apsusc.2014.04.189.

[36] S. Sharma, Density functional calculation of silicon adatom adsorption on pure and defected graphene, Philos. Mag. 94 (2014) 867-875, doi:http://dx.doi.org $10.1080 / 14786435.2013 .861945$.

[37] K. Takeda, K. Shiraishi, Theoretical possibility of stage corrugation in Si and Ge analogs of graphite, Phys. Rev. B. 50 (1994) 14916-14922, doi:http://dx.doi. org/10.1103/PhysRevB.50.14916.

[38] E.P.M. Leiva, G.L. Luque, M.I. Rojas, Curvature effect in the longitudinal unzipping carbon nanotubes, J. Solid State Electrochem. 17 (2013) 1189-1200, doi:http://dx.doi.org/10.1007/s10008-012-1992-0.

[39] A. Lopez-bezanilla, W. Zhou, J. Idrobo, Electronic and Quantum Transport Properties of Atomically Identified Si Point Defects in Graphene, J. Phys. Chem. Lett. 5 (2014) 1711-1718, doi:http://dx.doi.org/10.1021/jz500403h.

[40] Y. Tang, Z. Yang, X. Dai, A theoretical simulation on the catalytic oxidation of CO on Pt/graphene, Phys. Chem. Chem. Phys. 14 (2012) 16566-16572, doi:http:/ dx.doi.org/10.1039/c2cp41441d.

[41] G. Eranna, CRYSTAL GROWTH AND EVALUATION OF SILICON for VLSI and ULSI, CRC, 2015, 2016.

[42] C. Bena, S.A. Kivelson, Quasiparticle scattering and local density of states in graphite, Phys. Rev. B. 72 (2005) 1-5, doi:http://dx.doi.org/10.1103/ PhysRevB.72.125432.

[43] W. Zhang, P. Zuo, C. Chen, Y. Ma, X. Cheng, C. Du, et al., Facile synthesis of binder-free reduced graphene oxide/silicon anode for high-performance lithium ion batteries, J. Power Sources 312 (2016) 216-222, doi:http://dx.doi. org/10.1016/j.jpowsour.2016.01.106.

[44] C.M. Zhao, M.C. Hayner, In-Plane Vacancy-Enabled High-Power Si -Graphene Composite Electrode for Lithium-Ion Batteries, Adv. Energy Mater. 1 (2011) 1079-1084, doi:http://dx.doi.org/10.1002/aenm.201100426. 
[45] J.H. Wu, F. Hagelberg, Density functional studies of small silicon clusters adsorbed on graphite (0001) and diamond (100), Phys. Rev. B. 76 (2007) 1-9, doi:http://dx.doi.org/10.1103/PhysRevB.76.155409.

[46] S.P. Koenig, N.G. Boddeti, M.L. Dunn, J.S. Bunch, Ultrastrong adhesion of graphene membranes, Nat. Nanotechnol. 6 (2011) 543-546, doi:http://dx.doi. org/10.1038/nnano.2011.123.

[47] C.B. Robledo, M.I. Rojas, O. Camara, E.P.M. Leiva, First-principles studies concerning optimization of hydrogen storage in nanoporous reduced graphite oxide, Intenational J. Hydrog. Energy. 39 (2014) 4396-4403, doi:http://dx.doi. org/10.1016/j.ijhydene.2013.12.206.

[48] O.A. Oviedo, M.M. Mariscal, E.P.M. Leiva, On the occurrence of stable and supersaturated metastable states in metallic core-shell nanoparticles, Phys.
Chem. Chem. Phys. 12 (2010) 4580-4589, doi:http://dx.doi.org/10.1039/ b924348h.

[49] D.J. Eaglesham, A.E. White, L.C. Feldman, N. Moriya, Equilibrium Shape of Si, Phys. Rev. Lett. 70 (1993) 1643-1647, doi:http://dx.doi.org/10.1103/ PhysRevLett.70.1643.

[50] R.J. Jaccodine, Surface Energy of Germanium and Silicon, J. Electrochem. Soc. 110 (1963) 524-527, doi:http://dx.doi.org/10.1149/1.2425806.

[51] G.S.E. Budevski, W.J. Lorenz, Electrochemical Phase Fomation and Growth: An Introduction to the Initial Stages of Metal Deposition, Weinheim, VCH, 1996.

[52] W. Wan, Q. Zhang, Y. Cui, E. Wang, First principles study of lithium insertion in bulk silicon, J. Phys. Condens. Matter 22 (2010) 1-9, doi:http://dx.doi.org/ 10.1088/0953-8984/22/41/415501. 